\title{
The Field Effect and Mott Transistor Based on Vanadium Dioxide
}

\author{
M.A. Belyaev, A.A. Velichko, P.P. Boriskov, N.A. Kuldin, V. V. Putrolaynen and G.B. Stefanovitch
}

\begin{abstract}
The paper presents simulation results of the field control of phase metal-insulator transition in transistor structures based on vanadium dioxide. The calculations of the field and the electron density in the space charge region in the metallic (M) and semiconducting (S) phases are given. It is shown that the field control of the phase transition at room temperature in the forward direction (from S- to M-phase) inefficiency due to the strong screening of the space charge on the Debye length does not exceed two unit cell of $\mathrm{VO}_{2}$.
\end{abstract}

Index Terms - metal-insulator transition, vanadium dioxide, field effect transistor, space charge region

\section{INTRODUCTION}

M ETAL-OXIDE-SEMICONDUCTOR field-effect transistors (MOSFET) have been for a long time the key elements of modern electronics industry. For the purpose of a permanent integration enhancement, the size of MOSFET has been decreasing exponentially for over decades in compliance with Moore's Law, but nowadays, owing to the intrinsic restrictions, the further scaling of MOSFET devices either encounters fundamental limits or demands more and more sophisticated and expensive engineering solutions. Alternative approaches and device concepts are currently designed both in order to sustain an increase of the integration degree, and to improve the functionality and performance of electronic devices. Oxide electronics is one of such promising approaches which could enable and accelerate the development of information and computing technology. The behavior of d-electrons in transition metal oxides is responsible for the unique properties of these materials, causing strong electron-electron correlations, which play an important role in the mechanism of metal-insulator transition. The Mott transition in vanadium dioxide is specifically the phenomenon that researchers consider as a corner stone of

Manuscript received May 17, 2014; accepted June 17, 2014. Date of online publication: June 30, 2014

This work was supported by the Strategic Development Program of Petrozavodsk State University (2012 - 2016) and the RF Ministry of Education and Science as a part of state program in the scientific field, projects no. № 2014/154, № 3.757.2014/K.

A. A. Velichko (velichko@psu.karelia.ru), P. P. Boriskov (boriskov@psu.karelia.ru), N.A. Kuldin (kuldin@psu.karelia.ru), V. V. Putrolaynen (vputr@psu.karelia.ru) are with the Chair of Electronics and Electroenergetics, M.A. Belyaev (biomax89@yandex.ru) and G. B. Steafanovitch (gstef@psu.karelia.ru) are with the Chair of Information Measuring Systems and Physical Electronics. Department of Physical Engineering at Petrozavodsk State University, 185910 Petrozavodsk, Russia. oxide electronics, particularly, in its special direction known as a Mott-transition field-effect transistor (MTFET).

The metal-insulator transition (MIT) in vanadium dioxide can be initiated by the temperature change: the MIT from semiconducting (S) phase to the metal (M) phase occurs at the critical temperature $T_{t} \sim 340 \mathrm{~K}$, and the re-entrant $(\mathrm{M} \rightarrow \mathrm{S})$ MIT occurs at $\mathrm{T}_{\mathrm{t}^{\prime}} \sim 320-338 \mathrm{~K}\left(\mathrm{~T}_{\mathrm{t}^{\prime}}<\mathrm{T}_{\mathrm{t}}\right.$ due to hysteresis [1]). According to the Hall effect measurements, $\mathrm{VO}_{2}$ is a n-type semiconductor [2]. The electric field effect on the MIT in $\mathrm{VO}_{2}$ has been studied earlier in many works [3-9]. The actuality of such studies is conditioned both by the necessity of investigation of the influence of electronic effects on the MIT, and by a practical interest, i.e. for fabrication of transistor-like devices [10]. In spite of this, fabrication of a MTFET is an unsolved problem yet [11].

In this work we estimate the field effect on the conductivity of $\mathrm{VO}_{2}$-based planar structures with the Mott mechanism of MIT [1]. This mechanism is concentration-induced, and transition from the S- to M-phase occurs at some critical electron density $n \sim n_{c l}$. On the other hand, the $\mathrm{M} \rightarrow \mathrm{S}$ transition occurs at $n \sim n_{c 2}\left(n_{c 2} \approx n_{m} / 3\right.$, were $n_{m}$ is the equilibrium electron density in the M-phase) [12].

\section{RESULTS AND DISCUSSION}

We consider an experimental scheme (Fig. 1) for observations of the field control by the MIT. The field in the structure is created by applying a voltage bias $U_{S i}$ to $\mathrm{Si}$ substrate with respect to the metal electrodes. Such configuration has been described in [17], though the resistivity change at room temperature has not been convincingly registered in work [17].

\section{A. The transition from $S$ - to $M$-phase}

Initially, the $\mathrm{VO}_{2}$ film is in the S-phase at room temperature. Under the action of an external field, the space charge region (SCR) appears at the $\mathrm{VO}_{2}$ boundaries. The total voltage drop on the structure of $\mathrm{U}$ is the sum of the voltage drops on $\mathrm{SiO} 2$ and the sum of the voltage drop across the SCR in VO2. Because of the negative polarity of $\mathrm{U}_{\mathrm{Si}}$ on the bottom surface potential $\psi_{s u}>0$ appears in the $\mathrm{VO}_{2} \mathrm{SCR}$. This $\psi_{s u}$ forms a layer enriched by electrons (Fig. 1.b). 


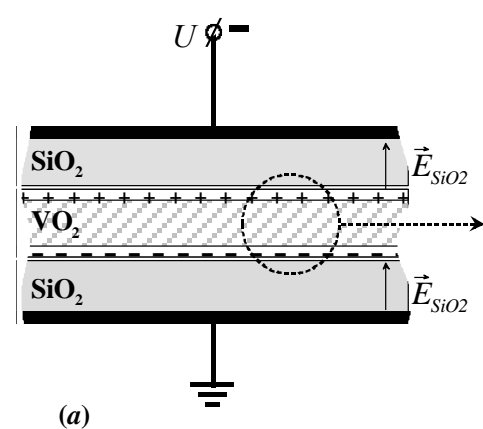

Fig.1. Experimental scheme for observation of the field effect inducing a Mchannel (a), SCR in $\mathrm{VO}_{2}$ at $n<n_{c l}$ (b), SCR in $\mathrm{VO}_{2}$ at $n>n_{c l}$ (c). $U_{0}$ is the voltage determining the measuring current $\left(U_{0} \ll<U_{S i}\right), R_{L}-$ load resistance, $U_{t}$ - voltage across the structure, $L_{d S}$ and $L_{d M}$ - the Debye length in the S- and M- phases, respectively; $E_{\mathrm{SIO}_{2}}$ is the field in the $\mathrm{SiO}_{2}$ film.

In order to calculate the electron density distribution in this region, one can use the Poisson equation [14]. As a result, for a n-type semiconductor under the condition $\left(\frac{d \psi}{d z} \rightarrow 0\right.$, at $\psi \rightarrow 0$ ), we obtain the expression for the field $E$ :

$$
E=\left(\frac{d \psi}{d z}\right)=\frac{\sqrt{2}}{\beta \cdot L_{d}}\left(e^{\beta \psi}-\beta \psi-1\right)^{1 / 2}
$$

where $\psi$ is the potential, $\beta=\frac{q}{k T}, q$-electron charge, $k$ - the Boltzman constant, $T$ - temperature, $L_{d}=L_{d S}$ - the Debye length in the S-phase. The latter is calculated as:

$$
L_{d}=\sqrt{\frac{\varepsilon \cdot \varepsilon_{0}}{\beta \cdot q \cdot n_{0}}}
$$

with $n_{0}=n_{s}$ being the equilibrium electron density in the $\mathrm{VO}_{2}$ S-phase, $\varepsilon=\varepsilon_{s}-$ the dielectric permittivity, and $\varepsilon_{0}$ - the dielectric constant.

It is impossible to obtain an analytical expression for $\psi(z)$. Therefore Eq. (1) was integrated numerically with the following boundary conditions: $z=0, \psi=\psi_{s u} ; z=\mathrm{z}, \psi=\psi$. As the result of calculations, the dependences of $E(z)$ and $n(z)$ were obtained (Fig. 2), where

$$
n(z)=n_{0} \cdot e^{\beta \psi(z)}
$$

It should be noted that the surface field $E(0)=E_{S I O 2}$, since $E(z)$ is the external field dependence on the distance from the surface, which drops due to the screening effect.

The $\psi_{s u}$ values were chosen so that the critical density was achieved in a $9 \AA$-thick layer $\left(\sim\right.$ i.e. of the order of two $\mathrm{VO}_{2}$ unit cell dimension [1]), which can lead to the $\mathrm{S} \rightarrow \mathrm{M}$ MIT. The magnitude of the field at the $\mathrm{SiO}_{2}-\mathrm{VO}_{2}$ boundary at this point is $E_{c l} \sim 1.6 \cdot 10^{5} \mathrm{~V} \cdot \mathrm{cm}^{-1}$ (Fig. 2.a).
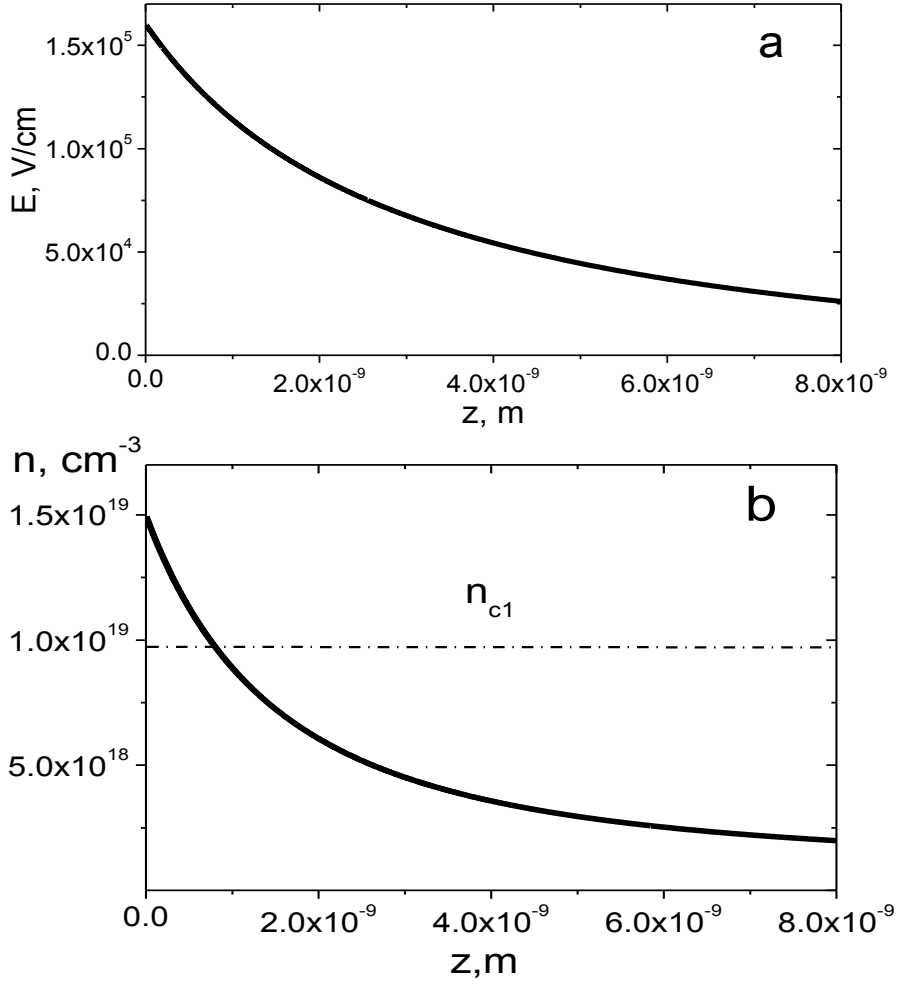

Fig.2. Electric field strength distribution $E(z)-$ (a) and concentration distribution $n(z)-(\mathrm{b})$ in $\mathrm{VO}_{2} \mathrm{SCR} ; T=300 \mathrm{~K}, \varepsilon_{s}=41$ [15], $n_{s}=10^{18} \mathrm{~cm}^{-3}, \psi_{\mathrm{su}}$ $=0.07 \mathrm{~V}, n_{c l}=9.7 \cdot 10^{18} \mathrm{~cm}^{-3}[16], \mathrm{L}_{\mathrm{dS}}=7.66 \cdot 10^{-9} \mathrm{~m}$.

Now we analyze what happens if a $9 \AA$ - thick layer undergoes the transition into the M-phase. In the formed Mlayer, the external field penetrates to a value of $\sim L_{d M}$ (Fig. 1.c). Using Eq. (2) and the values $\varepsilon=\varepsilon_{\infty}=9, n_{0}=n_{m}=$ $3.3 \cdot 10^{22} \mathrm{~cm}^{-3}$ for the M-phase of $\mathrm{VO}_{2}$ [12] at $T=300 \mathrm{~K}$ we obtain $L_{d M} \sim 2 \cdot 10^{-11} \mathrm{~m}$. Thus, the formed metal layer screens the external field completely. Because of the ohmic contact between the M- and S-phase, there is no electric field behind the metal layer, and hence the SCR in the S-phase eliminates (Fig. 1.b.c). Further increase of the field up to the $\mathrm{SiO}_{2}$ breakdown $\left(\sim 1.3 \cdot 10^{7} \mathrm{~V} \cdot \mathrm{cm}^{-1}[14]\right)$, will not result in a significant change of the situation outlined (Fig. 3.a).

As can be seen from the figure 3 the field inside the $\mathrm{M}$ phase falls down to $E_{c l}$ at a distance of $\sim 0.8 \AA$. If we use the value $n_{m}=1 \cdot 10^{21} \mathrm{~cm}^{-3}$ (metallic phase of $\mathrm{VO}_{2}$ can possess such electron density due to nonstoichiometry [1]), this distance will be $\sim 4 \AA$ which again does not exceed one cell parameter.

Theoretically, a transition of a $5 \AA$-thick layer of $\mathrm{VO}_{2}$ in the M-phase, in a $\sim 1000 \AA$-thick film, will lead to the decrease of the longitudinal resistance by a factor of six $\left(\rho_{M}=0.001 \Omega \cdot \mathrm{cm}\right.$, $\rho_{S}=1 \Omega \cdot \mathrm{cm}$ are the specific resistivities of the M- and Sphases [1]). In reality, the effect would be much weaker because of roughness of the substrate and defects in the film.

One can offer the scheme (Fig. 4) for observation of this effect, where the field is created by applying a bias voltage to the $\mathrm{U}_{\mathrm{Si}}$ Si-substrate relative to metallic electrodes. This configuration has been described in [13], however, change in 
resistance was relatively low what is apparently explained by the above arguments.
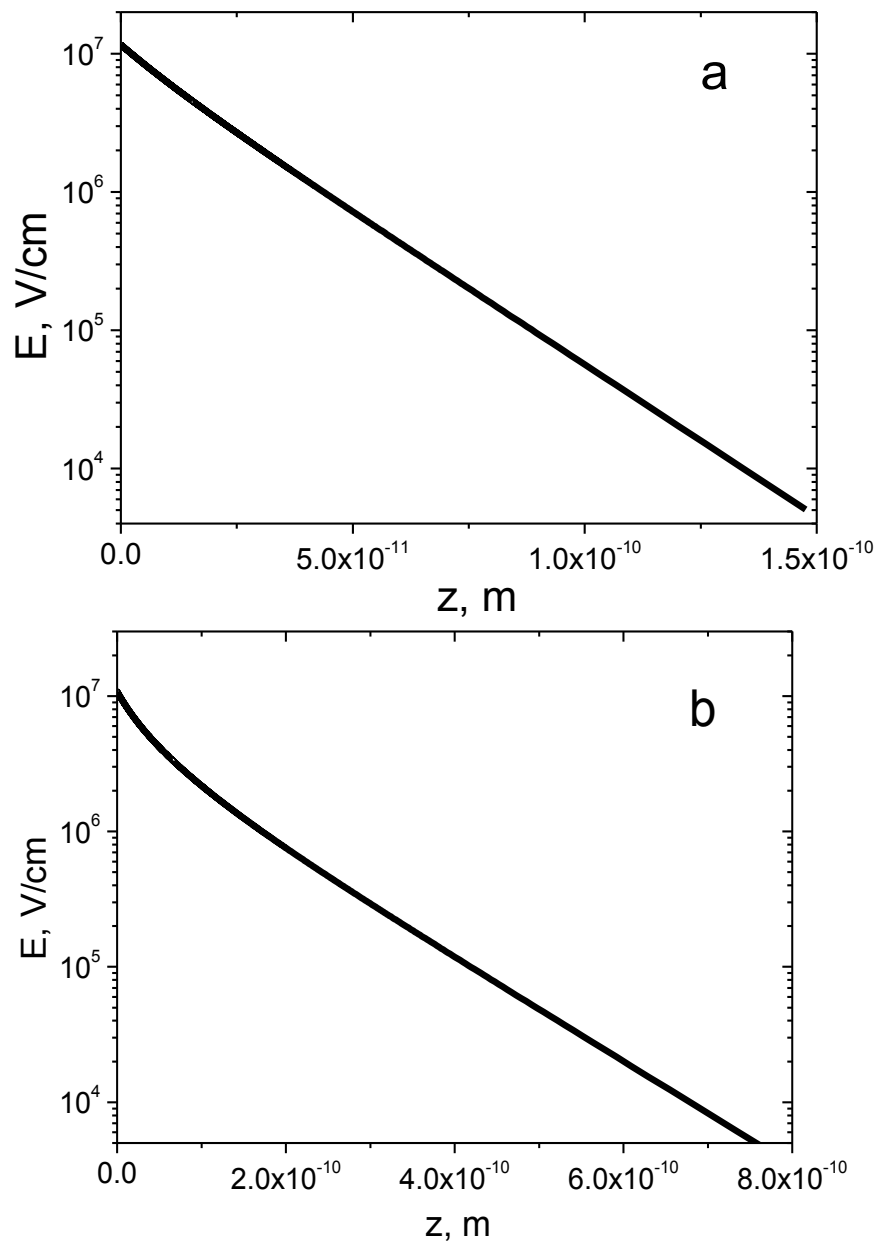

Fig.3. Electric field strength distribution in M-phase (enrichment case): $n_{m}=$ $3.3 \cdot 10^{22} \mathrm{~cm}^{-3}-$ (a), $n_{m}=1 \cdot 10^{21} \mathrm{~cm}^{-3}-$ (b), $T=300 \mathrm{~K}, E_{\text {ext }} \sim 1 \cdot 10^{7} \mathrm{~V} \cdot \mathrm{cm}^{-1}$.

Even if the contribution of the formed M-layer to the total resistance could be fixed experimentally, the transistor effect would be absent, because as the field is further increased, as it was said above, it penetrates on a depth not larger than the size of a unit cell, which would not alter the total resistance too much.

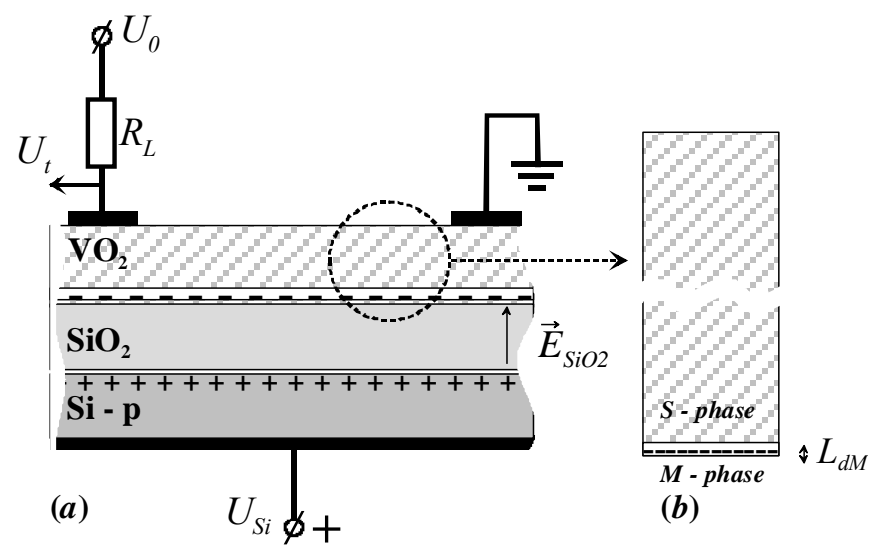

Fig.4. Experimental scheme for observation of the field effect inducing a Mchannel, $U_{0}$ is the voltage determining the measuring current $\left(U_{0} \ll U_{S i}\right), R_{L}-$ load resistance, $U_{t}$ - voltage across the structure, $L_{d M}$ - the Debye length in the M- phase, $E_{\mathrm{SIO} 2}$ is the field in the $\mathrm{SiO}_{2}$ film.
The structure would therefore operate as an electronic key or switch. It is to be noted that the above-described results are valid in a static regime, $U_{S i}=$ const. A pulse regime can lead to the appearance of shift currents resulting in new effects influenced upon the MIT. For example, the boundary temperature change due to either the Joule heat or the Peltier effect at the $\mathrm{M}-\mathrm{S}$ boundary in $\mathrm{VO}_{2}$ [1] could contribute in this case.

\section{B. The transition from $M$ - to S-phase}

Next we analyze a possible field effect on the re-entrant $(\mathrm{M} \rightarrow \mathrm{S})$ MIT. A scheme similar to that shown in Fig. 1.a, was considered, but the ambient temperature was maintained above $T_{t}(\sim 345 \mathrm{~K})$, and $\mathrm{n}$-type $\mathrm{Si}$ with the bias voltage $U_{S i}<0$ was used.

In this structure, $\mathrm{VO}_{2}$ is initially in M-phase. Under the field on the border of $\mathrm{VO}_{2}-\mathrm{SIO}_{2} \mathrm{SCR}$ forms depleted layer (Fig. 5) and accumulates positive charge of ionized cores of lattice. In this case, the field distribution has a somewhat different character, as high fields can penetrate a greater distance than in the case of enrichment. We prove this by bringing the concentration distribution $n(z)$ and field $E(z)$. Formulae for calculation similar to formulas (1) and (2) except that $\psi_{s u}<0$ and (1) shows the "minus" to the whole expression for the change of the field direction, and $\mathrm{T}=345 \mathrm{~K}$.

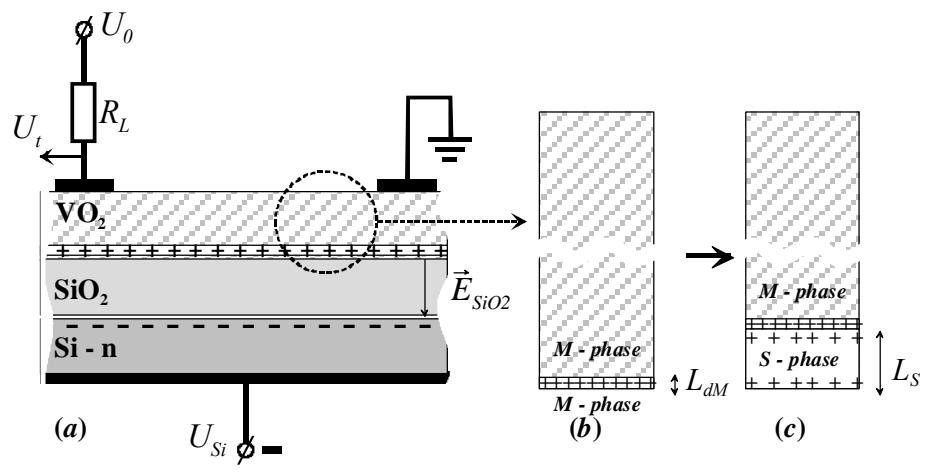

Fig.5. Experimental scheme for observation of the field effect inducing a Schannel, $U_{0}$ is the voltage determining the measuring current $\left(U_{0} \ll U_{S i}\right), R_{L}-$ load resistance, $U_{t}$ - voltage across the structure, $L_{d M}$ - the Debye length in the M- phase, $E_{\mathrm{SIO} 2}$ is the field in the $\mathrm{SiO}_{2}$ film.

As Fig. 6.a shows the critical concentration $n_{c 2}$ of reverse MIT can not be achieved even at high field $\left(\sim 1 \cdot 10^{7} \mathrm{~V} \cdot \mathrm{cm}^{-1}\right)$ for value $n_{m}=3.3 \cdot 10^{22} \mathrm{~cm}^{-3}$. If we assume, as we did above, that $n_{\mathrm{m}}=1 \cdot 10^{21} \mathrm{~cm}^{-3}$, then $n_{c 2}$ is achieved at distances of about the same size of $\mathrm{VO}_{2}$ unit cell (Fig. 6.c). Thus, comparing with the Fig. 2.a (the enriched regime), where the field decreases to critical value $E_{c}$ at distance $9 \mathrm{~A}$ instead of $5 \mathrm{~A}$ as the case of depletion regime.

We obtain that at $n_{m}=1 \cdot 10^{21} \mathrm{~cm}^{-3}$ and $E \sim 1 \cdot 10^{7} \mathrm{~V} \cdot \mathrm{cm}^{-1}$ it is feasible to reach the density $n_{c 2}$ and thereby to transform a $\sim 5$ $\AA$-thick layer to the S-phase. In this case, the electron density in this layer would fall, and the field is now applied to the S-M boundary; then a next layer will undergo the transition into the S-phase. As the result of this self-spreading wave of the S- 
phase (“domino effect"), a significant portion of the metal film might transit into the $\mathrm{S}$-phase.
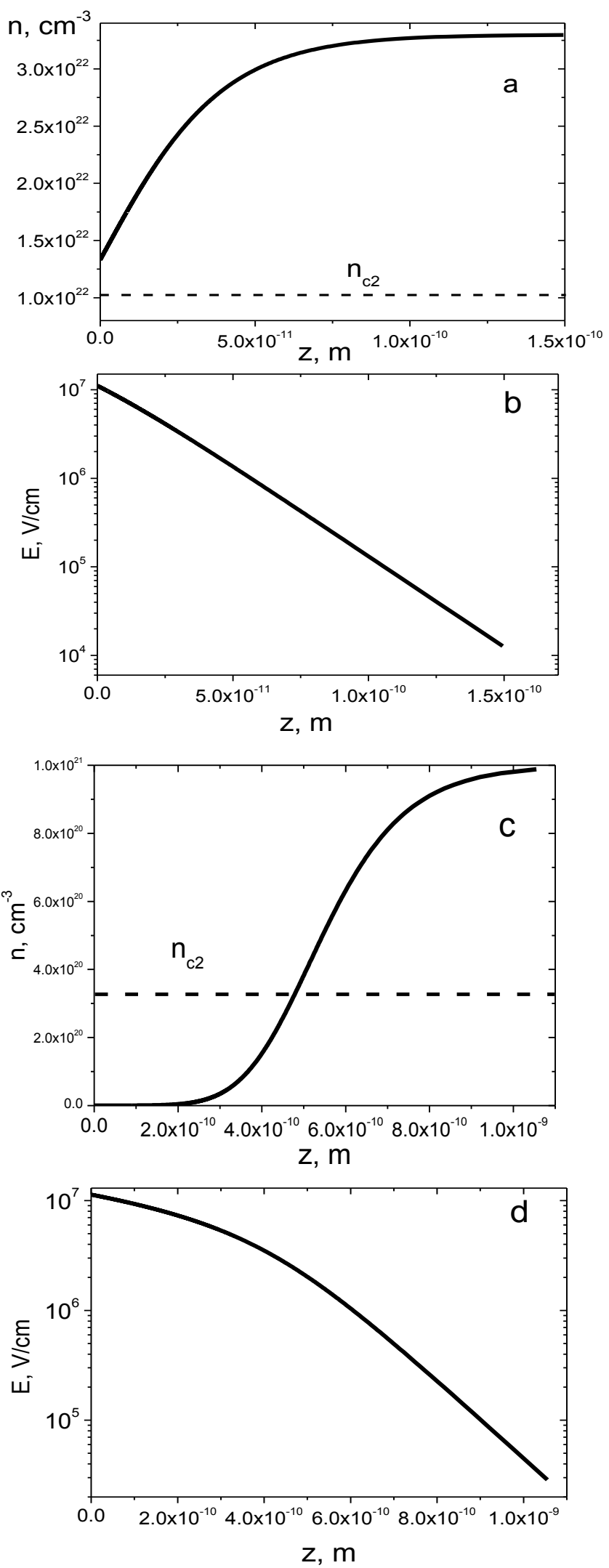

Fig.6. Electric field strength distribution $E(z)$ and concentration distribution $n(z)$ in M-phase (depletion case): $n_{m}=3.3 \cdot 10^{22} \mathrm{~cm}^{-3}-\left(\mathrm{a}\right.$ and b), $n_{m}=1 \cdot 10^{21}$ $\mathrm{cm}^{-3}-\left(\mathrm{c}\right.$ and d), $T=345 \mathrm{~K}\left(n_{c 2} \approx n_{m} / 3\right) ; E_{e x t} \sim 1 \cdot 10^{7} \mathrm{~V} \cdot \mathrm{cm}^{-1}$.
The thickness of the layer is determined by voltage $\mathrm{U}_{\mathrm{Si}}$, which is distributed between $\mathrm{SiO}_{2}$ and has been converted to $\mathrm{S}$-phase layer of $\mathrm{VO}_{2}$. Calculate how the field is distributed in the resulting layer of $\mathrm{S}$-phase. If we assume that the equilibrium concentration is $n_{0}=n_{0}(345 \mathrm{~K}) \sim n_{c l}$ and external field is $E_{\text {ext }} \sim 1 \cdot 10^{7} \mathrm{~V} \cdot \mathrm{cm}^{-1}$, the form $n(z)$ and $E(z)$ is represented in figure 7.ab.
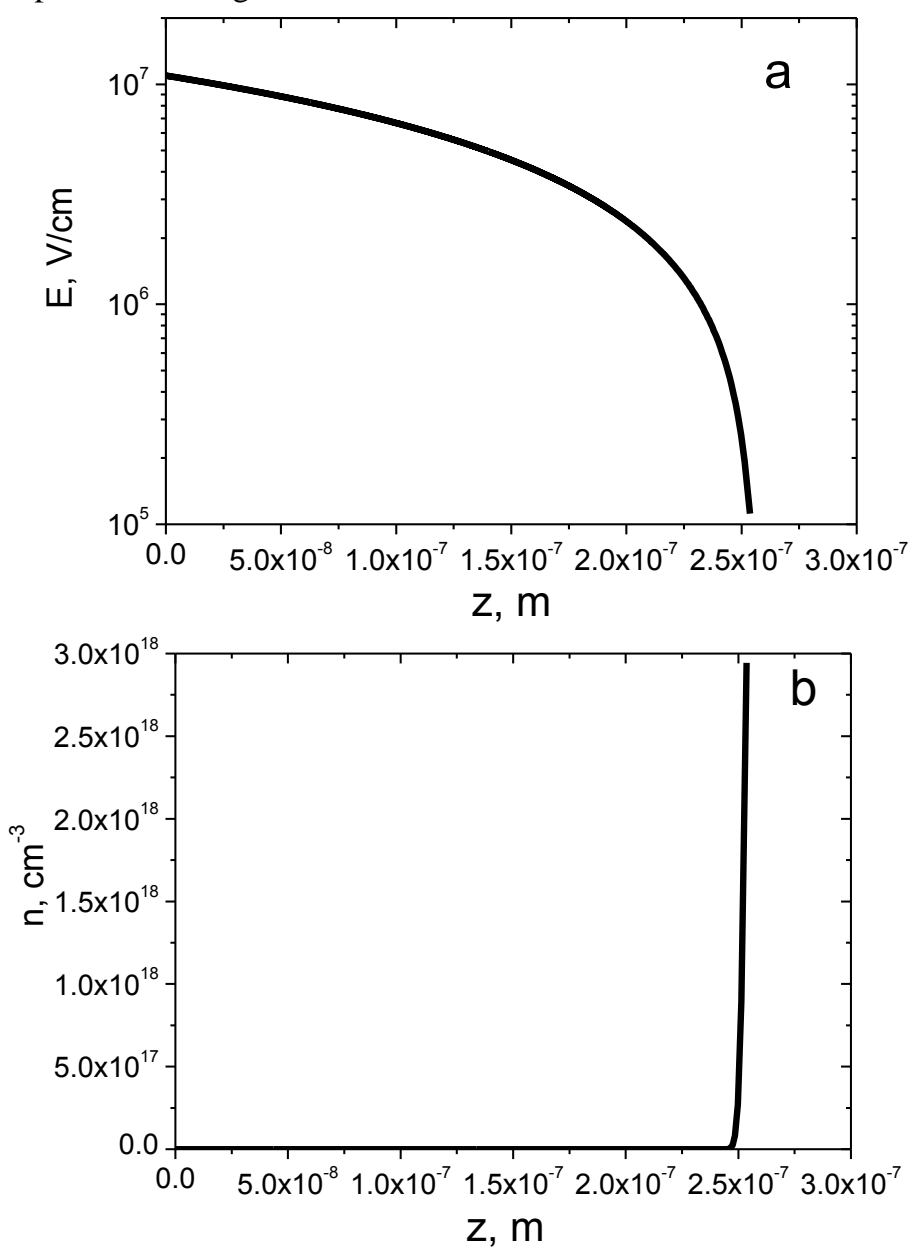

Fig.7. Electric field strength distribution $E(z)$ and concentration distribution $n(z)$ in M-phase (depletion case), $E_{\text {ext }} \sim 1 \cdot 10^{7} \mathrm{~V} \cdot \mathrm{cm}^{-1}$.

As Fig. 7. shows the screening of the field occurs only at a distance of $\sim 2500 \mathrm{~A}$, at distances $\sim 1000 \mathrm{~A}$ field can be considered constant in the absence of free carriers. Thus, the $\mathrm{VO}_{2}$ films 1000A thick to form a layer S-phase can be considered a dielectric.

However, taking into account the high-field effects (e.g. Poole-Frenkel effect), the electron density in the S-phase can be estimated:

$$
n_{0}=n_{0}(345 K) \cdot e^{\frac{b m \cdot \sqrt{E}}{2 \cdot k T}}
$$

where $b m=\sqrt{\frac{q^{3}}{\pi \cdot \varepsilon_{S} \cdot \varepsilon_{0}}}-$ the Poole-Frenkel constant.

Substituting $E \sim 1 \cdot 10^{7} \mathrm{~V} \cdot \mathrm{cm}^{-1}$, we obtain $n \sim 5.8 \cdot 10^{21} \mathrm{~cm}^{-3}$ which gives the distribution $n(z)$ and $E(z)$ (Fig.8.ab). The field seen can be considered constant thickness to $\sim 100 \mathrm{~A}$. Thus, 
the channel width of the dielectric can be adjusted to 100A. What can be quite appreciable adjustment current passing through the $\mathrm{VO}_{2}$ channel, with a thickness of up to $1000 \mathrm{~A}$ and above.
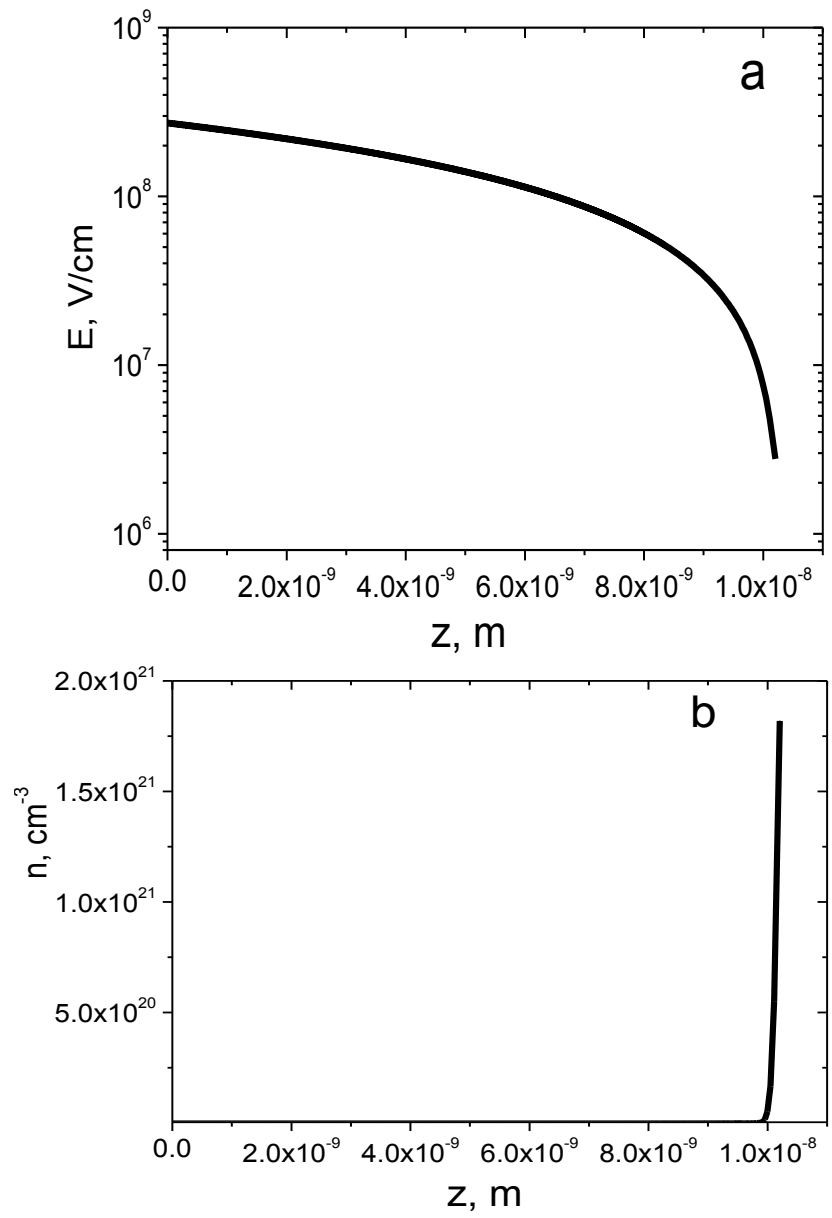

Fig.8. Electric field strength distribution $E(z)$ and concentration distribution $n(z)$ in M-phase considering Poole-Frenkel effect (depletion case), $E_{\text {ext }} \sim 1 \cdot 10^{7}$ $\mathrm{V} \cdot \mathrm{cm}^{-1}$.

As the external fields can cause breakdown of $\mathrm{SiO}_{2}$, leading simultaneously to the breakdown of the entire structure.

\section{CONCLUSION}

Thus, the simulation results showed a slight penetration of the field in S- and in M-phase of $\mathrm{VO}_{2}$, what greatly reduces the field control efficiency of MIT. This is particularly evident in the case of transition from S- to M-phase, where the strong screening of the space charge gives the Debye length does not exceed two unit cell of $\mathrm{VO}_{2}$. An alternative method to enhance the field effect can be a decrease of temperature in the structure, when the relative change of $\mathrm{VO}_{2}$ channel resistance increases with the growth of the total resistance as shown in $[13,18]$.

\section{REFERENCES}

[1] N. F. Mott, Metal-Insulator Transitions. London: Taylor \& Francis, 1990.

[2] A.A. Bugaev, B.P. Zaharchenya, and F.A. Chudnovskii, MetalSemiconductor Phase Transition and It Application. Leningrad: Nauka, 1979, p. 184.

[3] D. Ruzmetov, G. Gopalakrishnan, C. Ko, V. Narayanamurti, and S. Ramanathan, "Three-terminal field effect devices utilizing thin film vanadium oxide as the channel layer," J. Appl. Phys., 107, p. 114516, 2010.

[4] H. T. Kim, B. G. Chae, D. H. Youn, S. L. Maeng, G. Kim, K.-Y. Kang, and Y.-S. Lim, "Mechanism and observation of Mott transition in VO2based two- and three-terminal devices," N. J. Phys., 6, p. 52, 2004.

[5] S. Hormoz and S. Ramanathan, "Limits on vanadium oxide Mott metalinsulator transition field-effect transistors," Solid-State Electron., 54, pp. 654-659, 2010.

[6] C. Zhou, D. M. Newns, J. A. Misewich, and P. C. Pattnaik, "A field effect transistor based on the Mott transition in a molecular layer," Appl. Phys. Lett., 70, pp. 598-600, 1997.

[7] B. Wu, A. Zimmers, H. Aubin, R. Ghosh, Y. Liu, and R. Lopez, "Electric-field-driven phase transition in vanadium dioxide," Phys. Rev. $B, 84$, p. 241410, 2011.

[8] S. Sengupta, K. Wang, K. Liu, A. K. Bhat, S. Dhara, J. Wu, and M. M. Deshmukh, "Field-effect modulation of conductance in $\mathrm{VO}_{2}$ nanobeam transistors with $\mathrm{HfO}_{2}$ as the gate dielectric," Appl. Phys. Lett., 99, p. 062114, 2011.

[9] A. L. Pergament, P. P. Boriskov, A. A. Velichko, and N. A. Kuldin, "Switching effect and the metal-insulator transition in electric field," $J$. Phys. Chem. Solids, 71, pp. 874-879, 2010.

[10] Z. Yang, C. Ko, and S. Ramanathan, "Oxide Electronics Utilizing Ultrafast Metal-Insulator Transitions," Апnи. Rev. Mater. Res., 41, pp. 337-367, 2011.

[11] Y. Zhou and S. Ramanathan, "Correlated Electron Materials and Field Effect Transistors for Logic: A Review," Crit. Rev. Solid State Mater. Sci., 38, 4, pp. 286-317, 2013.

[12] A. Pergament, E. Kazakova, A. Morak, "The photoinduced Mott transition from metal to insulator: the problem of critical concentration," J. Phys.: Condens. Matter, 17, 7, p. 1151, 2005.

[13] A. A. Velichko, V. V. Putrolaynen, M. A. Belyaev, G. B. Stefanovich, A. L. Pergament Field-effect modulation of resistance in $\mathrm{VO}_{2}$ thin film at lower temperature, Jap. J. of Appl. Phys., to be published.

[14] S. M. Sze, Physics of Semiconductor Devices. NY: Wiley, 1981.

[15] Z. Yang, C. Ko, V. Balakrishnan, G. Gopalakrishnan, S. Ramanathan, "Dielectric and carrier transport properties of vanadium dioxide thin films across the phase transition utilizing gated capacitor devices," Phys. Rev. B, 82, 20, p. 205101, 2010.

[16] A. S. Barker, H. W. Verleu, and H. J. Guggenheim, "Infrared optical properties of vanadium dioxide above and below transition temperature," Phys. Rev.Lett, 17, 26, p. 1286, 1966.

[17] V. V. Mokrousov and V. N. Kornetov, "Field effects in vanadium dioxide films," Fiz. Tv. Tela [Sov. Phys. Solid State], 16, 10, pp. 3106-3107, 1974.

[18] A. L. Pergament, G. B. Stefanovich, A. A. Velichko, "Oxide Electronics and Vanadium Dioxide Perspective: A Review", Journal on Selected Topics in Nano Electronics and Computing, vol. 1, pp. 24-43, 2013. 